Giraldo, C.; Sinisterra, E.; Arteaga, M. (2018). Prácticas contables generalmente utilizadas en el sector informal: Caracterización de las microtiendas de la comuna cuatro de Medellín. Contaduría Universidad de Antioquia, 72, 59-80 Doi: https://doi.org/10.17533/udea.rc.n72a04

\title{
Prácticas contables generalmente utilizadas en el sector informal: Caracterización de las microtiendas de la comuna cuatro de Medellín ${ }^{*}$
}

\author{
Carolina Giraldo Ruiz $^{* *}$ \\ carolina.giraldor@udea.edu.co \\ Universidad de Antioquia \\ Estefany Sinisterra Rincón \\ estefany.sinisterra@udea.edu.co \\ Universidad de Antioquia \\ Michelle Arteaga Londoño \\ michelle.arteaga@udea.edu.co \\ Universidad de Antioquia
}

Este artículo se deriva del trabajo de grado para optar al título profesional otorgado por la Universidad de Antioquia, Facultad de Ciencias Económicas, Departamento de Ciencias Contables.

** Autor para correspondencia. 
Prácticas contables generalmente utilizadas en el sector informal: Caracterización de las microtiendas de la comuna cuatro de Medellín

Resumen: Mediante una metodología de investigación con enfoque cualitativo e inductivo, se desarrollan las características que el grupo investigador logra explorar sobre las prácticas contables generalmente utilizadas por los tenderos en los microestablecimientos de la comuna cuatro de Medellín, con el objetivo de obtener y consolidar información concreta y empírica del mencionado sector, para transitar de la intuición a un conocimiento más certero y riguroso y ser base para posteriores desarrollos de un sector que tiene gran incidencia a nivel nacional. En pro de esto y para lograr un conocimiento contextual del objeto de estudio, se realiza una descripción del sector económico informal, además de otorgar varias definiciones de corte económico, legal y contable inherentes a la temática. Finalmente, se concluye que las tiendas de barrio de la comuna cuatro no implementan prácticas contables tan formales y rigurosas como las propuestas en las NIIF Simplificadas, sino, que por el contrario, por tradición, comodidad y facilidad, este sector realiza una serie de prácticas que aunque carecen de soporte técnico, resultan ser suficientes y estar alineadas a sus necesidades. Adicionalmente, se realiza un llamado a enaltecer el carácter social de la contaduría pública frente a las situaciones más cotidianas de la sociedad en las cuales se aplique el saber.

Palabras Clave: Economía popular, microtiendas, tenderos, prácticas contables generalmente aceptadas, contabilidad informal.

Accounting practices generally used in the informal sector: characterization of the micro stores in District Four of Medellín

Abstract: Through a research methodology with a qualitative and inductive focus, this research explored and defined the characteristics of the generally used accounting practices by store owners in the micro establishments of District Four of Medellin, with the aim of obtaining and consolidating concrete and empirical information of said sector, to move from intuition to a more ascertained and rigorous knowledge so as to be the basis for subsequent developments in a sector with great incidence at national level. To do this, and to achieve contextual knowledge of the study object, a description of the informal economic sector is made, and several definitions of economic, legal and accounting nature inherent to the topic are provided. Finally, it is concluded that neighborhood stores in District Four will not implement accounting practices as formal and rigorous as the ones proposed by the Simplified IFRS. On the contrary, for reasons of traditions, comfort and easiness, stores in this sector carry out a series of practices that, although lacking technical foundations, prove to be sufficient and are aligned with their needs. Additionally, there is a call to exalt the social nature of Public Accounting in the habitual situations of society in which knowledge is applied.

Keywords: Popular economy, micro stores, store owners, generally accepted accounting practices, informal accounting.

Les pratiques comptable généralement utilisés dans le secteur informel : caractérisation des microsupérettes de la Commune quatre de Medellín.

Résumé: En faisant appel à une méthodologie de recherche d'une approche qualitative et inductive, le groupe de recheche réussi à explorer les caracteristiques des pratiques comptables appliquées par les propriétaires des microétablissement de commerce de la commune quatre de Medellín. Ceci afin d'obtenir et renforcer l'information spécifique et impirique de ce secteur pour aller de l'intuiton vers une connaissance plus rigoureuse et précise, ainsi que de servir de base à des devéloppements ultérieurs d'un secteur d'une grande incidence en Colombie. Afin de parvenir à une connaissance contextuelle de l'objet d'étude, une description du secteur économique informelle a été faite et plusieurs définitions à caractère économique, legal et comptable liées au sujet ont éte prises en compte. Finalement, l'article conclut que les supérettes de quartier de la commune quatre de Medellín ne suivent pas de pratiques aussi formelles et strictes que celles proposées par las IFRS simplifiées. Au contraire, par tradition, practicité et simplicité, le secteur suit une série de pratiques qui s'averent être suffisantes et alignées aux besoins malgré leur manque de support technique. En plus, un appel est fait à exalter le caracter social de la comptabilité publique face aux situations les plus quotidiennes de la société dans lesquelles ce savoir est appliqué.

Mots-clés: Économie populaire, minsupérettes, petit commercants, pratiques comptables généralement acceptées, comptabilité informelle.

Práticas contábeis geralmente usadas no setor informal: caracterização das mercearias da comuna quatro de Medellin.

Resumo: Através de uma metodologia de pesquisa com foco qualitativo e indutivo, se desenvolvem as características que o grupo pesquisador consegue explorar sobre as práticas contábeis, geralmente utilizadas pelos comerciantes das pequenas mercearias da comuna quatro de Medellin, com o intuito de obter e consolidar informação concreta e empírica deste setor, para passar da intuição a um conhecimento com maior certeza e rigorosidade e ser uma base para futuros desenvolvimentos de um setor que tem grande incidencia no âmbito nacional. Em prol disto e para atingir um conhecimento contextual do objeto de estudo, se realiza uma descrição do setor econômico informal, além de providenciar várias definições do tipo econômico, legal e contábil inerentes ao assunto. Finalmente, conclui-se que as mercearias da comuna quatro não adotam práticas contábeis tão formais e rigorosas como as propostas nas NIIF Simplificadas, mas pelo contrario, por tradição, conforto e facilidade, este setor realiza uma série de práticas que, mesmo que não têm suporte técnico, são suficientes e estão alinhadas com as suas necessidades. Adicionalmente, se faz um chamado a exaltar o caráter social da contabilidade pública frente às situações mais cotidianas da sociedade nas quais se aplique o saber.

Palavras chave: Economia popular, mercearias, práticas contábeis geralmente aceites, contabilidade informal. 
Cont. udea (enero-junio), pp. 59-80. (C) Universidad de Antioquia-2018.

\title{
Prácticas contables generalmente utilizadas en el sector informal: Caracterización de las microtiendas de la comuna cuatro de Medellín
}

\author{
Carolina Giraldo Ruiz, Estefany Sinisterra Rincón y Michelle Arteaga Londoño
}

Doi: https://doi.org/10.17533/udea.rc.n72a04

Primera versión recibida en mayo de 2018- Versión final aceptada en junio de 2018

\section{Introducción}

I as microtiendas son establecimientos que aún con su clasificación Lreduccionista, tienen una gran participación en la economía y generación de empleo a nivel nacional debido a su masiva existencia, así como lo establece la afirmación de que las tiendas de barrio, ocupan el primer lugar dentro del sector informal, atendiendo a que en el país, y especialmente en el municipio de Medellín, su número es superior al de otro tipo de establecimientos o negocios en esta y otras ciudades. (Días, 2014) Sin embargo, son negocios cuya caracterización se ha ahondado desde perspectivas económicas y antropológicas, más su estudio desde la práctica y la teoría contable ha sido mínimo o con resultados enfocados a la estadística, o por lo menos, esta es la percepción encontrada a partir de la consulta de antecedentes teóricos de la temática.

En este sentido surge la motivación del grupo investigador, que al querer realizar un proyecto de tinte social, identifica una ausencia de documentación y teoría contable acerca de las prácticas realizadas por los tenderos en pro del sostenimiento y manejo de los recursos, siendo esta necesaria para la formulación posterior de posibles ayudas a los terceros mencionados, como herramientas contables en aspectos dentro de los cuales esté el control de los recursos y la toma de decisiones, enfatizadas ciertamente al nivel de operación inmersa en la misma.

Como resultado de lo antes mencionado, se define caracterizar las prácticas contables generalmente utilizadas en las microtiendas ubicadas en la comuna cuatro de Medellín como objetivo de la presente investigación, con la intención 
Giraldo, Sinisterra, Arteaga. Prácticas contables generalmente utilizadas en el sector informal...

de llevar un proceso similar a realizado en la construcción inicial del marco técnico contable para las empresas. La metodología de estudio se desarrolla con enfoque cualitativo mediante una interacción presencial entre el grupo investigador y el objeto de estudio a través de entrevistas, instrumento cuya guía contiene puntos involucrados con el quehacer cotidiano del tendero respecto al manejo de los recursos, el conocimiento de conceptos contables o afines aplicados a sus negocios y el cumplimiento de obligaciones tributarias conforme a su categorización.

Para plasmar las situaciones y respuestas encontradas, se realizó una previa contextualización multidisciplinaria del objeto de estudio, en donde se define el sector y la relación entre los individuos que lo conforman; adicionalmente, se identifican las necesidades de información que manifiestan los tenderos y las formas y herramientas que usan como respuesta a las mismas. De este modo resulta posible una comparación respecto a la contabilidad concebida con todos los estándares técnicos actuales, y de algún modo evaluar la suficiencia de estas formas empíricas de controlar los recursos.

En pro de una reflexión profesional, se busca que todo lo desarrollado en la investigación sirva de manera propositiva a generar interés en los estudiantes y profesionales de contaduría pública frente a figuras cotidianas, que desde el saber contable podrían potencializarse y provocar un impacto social benéfico, que en este caso son aquellos cuya subsistencia se basa en la permanencia de microtiendas que están siendo afectadas por presiones económicas, tributarias y mercantiles, y para las cuales, mejorar el control de sus recursos y cumplir la amplia reglamentación del mercado formal, podrían ser variables vitales.

Atendiendo a la naturaleza de la investigación es necesario precisar que la misma se enmarca en un enfoque cualitativo e inductivo, dada la metodología bajo la cual se desarrollará el trabajo.

Inicialmente se puede decir que la contabilidad como ciencia social busca indagar el tejido en que se ven involucradas las tiendas de barrio, con el objetivo de describir e interpretar las prácticas contables internas y externas aplicadas por los gestores de las tiendas barriales para llevar a cabo un seguimiento de los recursos de la entidad, adicionando además que se aplica una metodología inductiva dado que a partir de la información proporcionada por cada uno de los tenderos, se pretende generalizar los resultados al total de la población entrevistada.

Finalmente es necesario detallar que además de lo anterior, la investigación toma una carácter cualitativo en el sentido que desde la planificación, se acude a métodos propios de este tipo para la selección y procesamiento de los principales aspectos del trabajo, así pues, el número de tenderos (muestra) se escoge a criterio de los investigadores, la recolección de la información se realizará a través de la entrevista, pues además de ser el medio ideal para 
captar la información requerida, dadas las características de la población objeto de la entrevista, es un instrumento en el que prima las expresiones discursivas y narrativas en los cuales se encierra un alto contenido subjetivo, más que la simple tabulación y muestra de datos.

La presente investigación tiene un alcance descriptivo atendiendo a las afirmaciones de Abreu (2012) que enuncia dicho alcance como aquel en que se busca responder al que, por qué, cuándo y cómo, para agrupar información de la población objeto de estudio y así, lograr un mayor conocimiento e interpretación de la misma.

De acuerdo con esto, se busca recopilar, organizar y analizar información relevante que posibilite identificar y caracterizar las prácticas contables llevadas a cabo actualmente en las tiendas de barrio de la comuna cuatro de la ciudad de Medellín. Al tener un alcance descriptivo, no sólo se alcanzan a identificar las características, tendencias y propiedades de las prácticas contables, sino que también se logran contextualizar en aquellas situaciones relacionadas e inmersas a su desarrollo y aplicación, permitiendo así un estudio más profundo del problema de investigación. Para ello, se recogen datos con base a hipótesis generadas a partir de la teoría, y se consolidan, para que de acuerdo con los resultados obtenidos, se construya las generalidades del objeto investigado; por lo que se deduce que la investigación utiliza el método inductivo para la generación del conocimiento.

Alcance que permite al grupo investigador evaluar la información resultante y plantear relaciones entre variables, que contextualicen la realidad de estos tipos de establecimiento dentro de la teoría contable. Dando de esta forma bases conceptuales para proyectos posteriores de construcción de herramientas, asesoramiento y de igual forma el continuo crecimiento de la teoría.

\section{Descripción del contexto}

Se ha tendido a relacionar el desarrollo de la sociedad con la industrialización, pero es una visión cuestionable, en el sentido de que los efectos colaterales que ha generado esta han sido la pobreza, desigualdad y desempleo; problemáticas a las que la sociedad ha construido alternativas de solución, sin mucho respaldo de las entidades gubernamentales, pero que han sido sostenibles y replicadas por las generaciones.

El desarrollo de una sociedad debe analizarse desde la capacidad de disminuir el desempleo y por ende la pobreza y la desigualdad en el campo laboral, la satisfacción inmediata de las necesidades y la reinversión de excedentes, características propias de la economía popular, también llamada, economía alternativa, economía autogestionaria, economía solidaria o empresas comunitarias, entendidas como el producto social frente a la imposibilidad del 
Giraldo, Sinisterra, Arteaga. Prácticas contables generalmente utilizadas en el sector informal...

sistema de brindar el derecho fundamental del trabajo digno para todos los colombianos. Según Montoya (2004) esta economía es un modelo alternativo, de y para las mayorías populares, fundamentado en su propio esfuerzo organizativo, definición corroborada con la de Molina y Restrepo (2012) quienes se refieren a la economía popular como las actividades económicas de producción de bienes y servicios, realizado por agentes individuales y colectivos que dependen de su fondo de trabajo propio, para el sostenimiento de su vida y la de sus familiares.

Se puede entonces definir la economía popular y social como iniciativas y actividades que el sector propone y desarrolla, con el objetivo de subsistir y alcanzar la satisfacción de sus necesidades económicas la cual, aunque inmersa en la informalidad, se puede considerar como un inductor de desarrollo social. Argumento apoyado por Razeto (2003) quien expresa que:

El sólo hecho de que varios miles de familias alcancen la auto subsistencia desde la situación marginal en que se encuentran y que lo hagan sin tener que recurrir a la acción asistencial del Estado o al sometimiento a las condiciones que les exige el capital (en otras palabras, sin depender de algún patrón), sería un logro formidable desde el punto de vista de crear las condiciones o premisas de un proceso de desarrollo auto sostenido. (Razeto, 2003, p. 48)

En este contexto, la economía popular genera desarrollo social en la medida en que emplea, en su mayor parte, a personas excluidas del mercado laboral, la actividad principal es satisfacer las necesidades de la sociedad, diversifica la oferta en el mercado y genera la subsistencia tanto del oferente como del demandante. En cuanto a esta última característica, se puede afirmar que es la causante de que esta economía sea una solución parcial a la situación actual, sin mucho crecimiento en el largo plazo. De acuerdo con lo anterior, "la economía popular no ha pasado de ser proyectos de autoabastecimiento para una familia o grupo, para quienes sobrevivir no va más allá de vivir en el día a día, con una economía de pobres para pobres, sin proyección social" (Moreno y Colorado, 1997); idea plasmada en la Ley de formalización y generación de empleo (Ley 1429,2010 ) en donde se reconoce que hay dos tipos de informalidades, la informalidad por subsistencia e informalidad con capacidad de acumulación, estando claramente nuestro trabajo de investigación dentro de la primera clasificación.

\section{II.1 Las microtiendas dentro del sector formal e informal}

Inicialmente es preciso conceptualizar lo que se entiende como economía informal para comprender las dinámicas que podrían enmarcarse dentro de esta definición. Así pues, la economía informal según el DANE (2017) citando a la OIT, se refiere a esta como actividades o unidades de trabajo dedicadas a la producción de bienes o prestación de servicios con la finalidad primordial 
de generar ingresos para las personas que participan en ella, sin cumplir todos los requerimientos formales establecidos por el Estado, lo anterior es complementado por el Banco de la República (2015), el cual menciona que además, tales actividades no cumplen con una serie de características administrativas y económicas con que debería cumplir una empresa formal. Por otro lado, y teniendo en cuenta lo citado previamente, podría delimitarse un concepto en lo que se refiere a la economía o sector formal, entendiéndose como aquella que rinde cuentas, es decir que además de tener el debido registro frente a las autoridades fiscales y comerciales pertinentes, conserva un orden administrativo basado en la realización de todas las actividades o procesos a los que se encuentre obligado.

Ahora bien, es preciso poner la lupa en las microtiendas, toda vez que son el objeto de la investigación. Abordando una mirada general, podría decirse que las tiendas de barrio son concebidas como unidades económicas que se clasifican en el sector informal, es decir, que se perciben como aquellas actividades que operan de manera desordenada en el sentido administrativo y que, además no cumplen con los deberes jurídicos a los que se estaría obligado, sin embargo, analizando cada uno de los puntos que se contraponen en los conceptos ya definidos y luego de estudiar los resultados obtenidos en la aplicación de una entrevista a tales establecimientos, se puede identificar que en general en las microtiendas converge una mezcla de tales variables, de tal forma que es posible clasificarla en ambos contextos.

Se iniciará por considerar el hecho de que la totalidad de las tiendas de barrio contempladas en las entrevistas están inscritas a Cámara de comercio y a la Dirección de Impuestos y Aduanas Nacionales (DIAN), por lo que al menos por este aspecto, podrían ser clasificadas en unidades que pertenecen al sector formal puesto que tributan lo correspondiente y en los periodos estipulados; no obstante, a la hora de discurrir en las actividades administrativas como llevar contabilidad, afiliación a la seguridad social, distinción del capital, entre muchos otros factores, se encuentra que se hace de manera parcial o en general no se hace, por lo que basados en estos aspectos, las microtiendas indiscutiblemente son unidades económicas que pertenecen a la informalidad, hecho que puede originarse en que el Estado si bien exige y promueve un registro en las debidas entidades, no vela por el cumplimiento de las obligaciones mínimas que se deben tener para garantizar el bienestar de la propia entidad, de los dueños y de los trabajadores.

Examínese brevemente ahora, que las tiendas de barrio ocupan el primer lugar dentro del sector informal, atendiendo a que tanto en el municipio de Medellín como en otras ciudades su número es superior al de otro tipo de establecimientos o negocios. En esta lógica, y por sus características 
Giraldo, Sinisterra, Arteaga. Prácticas contables generalmente utilizadas en el sector informal...

específicas, merece atención especial del gobierno tal como lo expresa la revista Cooperativismo y Desarrollo

El mundo empresarial popular, por su valor social y peso específico en la economía colombiana, debe merecer una especie de atención del gobierno, con el fin de incrementar su capacidad generadora de fuentes de trabajo, mejorar sus ingresos, y así elevar la calidad de más del $50 \%$ de la población Colombiana, actualmente marginada del desarrollo neoliberal, del crédito institucional y de las políticas económicas oficiales. (INDESCO, 2003, p. 111-119)

La anterior afirmación permite además contemplar aspectos como la financiación, de la cual son excluidas las microtiendas, por lo que sus opciones se ven reducidas primero a la banca, punto en el cual es preciso aclarar que las microtiendas y en general los establecimientos pertenecientes al sector informal, no son llamativos para las entidades financieras dado el riesgo y las bajas probabilidades de retorno, situación en mejora, de acuerdo con lo comentado por los tenderos respecto a entidades como el banco de las oportunidades y cooperativas barriales, las cuales están ampliando su cobertura; segundo, a familiares y personas naturales; y tercero, a elecciones más extremas como pagadiarios, de tal manera se confirma en Ramírez y Vergara (2014)

Los informales no son objeto prioritario de las entidades financieras por el riesgo que conllevan y las bajas esperanzas de éxito...solicitan dinero a familiares y amigos y otros manifiestan deber dinero a una persona natural y no a entidades bancarias (...) El uso del crédito gota a gota está generalizado en los microempresarios informales. (p. $35-42)$

\section{II.2 Microtiendas en el marco de la legalidad}

Con el objetivo de formalizar un negocio, hacer pública la calidad de comerciante, hacer visible la información del negocio frente a la sociedad y potenciales proveedores, y para facilitar la participación en la obtención de créditos ante entidades financieras, se ha optado por exigir a los comerciantes (personas naturales o jurídicas que desarrollen alguna actividad mercantil) la matricula en el registro mercantil y realizar su actualización anualmente, de igual forma, inscribir en este todos los libros, actas y documentos, acciones que si se incumplen conllevarán a multas económicas impuestas por la Superintendencia de Industria y Comercio (SIC) de hasta 17 Salarios Mínimos Mensuales Vigentes.

Como requisito previo para matricularse en el registro mercantil, se encuentra la expedición del Registro Único Tributario (RUT) con un Número de Identificación Tributaria (NIT) que permitirá la identificación del negocio frente a los demás establecimientos. El RUT deberá ser expedido, entre otros, por los responsables del impuesto sobre las ventas pertenecientes al régimen común o 
simplificado, estando las tiendas de barrio dentro de este último régimen por el hecho de vender productos gravados con el IVA de forma minorista, por contar con ingresos brutos inferiores a 3.500 UVT, tener máximo un establecimiento de comercio y cumplir con las demás características estipuladas en el artículo 499 del estatuto tributario modificado por la Ley 1819 en sus topes.

Adicional a la matrícula mercantil, dentro del mes siguiente al inicio de la actividad comercial, se debe inscribir en el municipio en que realiza la actividad mercantil, con el fin de declarar y pagar impuesto de industria y comercio de acuerdo a los ingresos percibidos por el comerciante. Cabe destacar, que si el contribuyente expide la matrícula mercantil en la cámara de comercio de Medellín, éste automáticamente queda inscrito en el municipio de Medellín para fines del ICA. Procedimiento que busca el anti trámite y la evasión de impuestos, en vista de que el contribuyente pudiera expedir la matrícula mercantil sin necesidad de inscribirse en el municipio para términos del impuesto de industria y comercio.

De acuerdo a los resultados observados en la aplicación del instrumento, se evidencia que la mayoría de tiendas de barrio cumplen con la obligación del pago al impuesto de industria y comercio y la renovación anual de la matrícula mercantil, siendo la expedición del RUT, un requisito previo para esta última acción. El cumplimiento a esta obligatoriedad se ejecuta, en unos casos al inicio del negocio, ya sea por conocimientos previos o por referencia de otros comerciantes; y en otros, por visitas domiciliarias realizadas por parte de funcionarios del municipio de Medellín para identificar comerciantes aptos a cumplir con la obligación de presentar y pagar ICA.

Es menester mencionar que, la única persona que no cumplía con el pago de ninguno de los dos impuestos mencionados anteriormente, estaba soportado por la norma en lo referente al impuesto de industria y comercio, en vista de que en el artículo 44 del Acuerdo Municipal de Medellín N 64 del 2012, se estipula que no están obligados a presentar la declaración de ICA, aquellos contribuyentes clasificados como régimen simplificado de industria y comercio, calidad que es adquirida por personas naturales, que ejerzan la actividad en un solo establecimiento o lugar físico, que el impuesto de ICA liquidado en el período respectivo no supere 28 UVT y que el contribuyente haya presentado las dos primeras declaraciones del impuesto de ICA desde el inicio de su actividad.

\section{II.2.1 Monotributo}

La reforma tributaria del 2016, a través del artículo 903 estipulado en el estatuto tributario nacional dio origen al monotributo, el cual no consiste en la creación de un nuevo tributo, sino que por el contrario, pretende la unificación 
de tres impuestos (renta, IVA y seguridad social), buscando la eficiencia tanto en el pago, como en el recaudo de los tributos.

Es un impuesto totalmente voluntario en el que el contribuyente puede elegir entre seguir pagando los impuestos como los venía pagando o hacerlo con el Monotributo; incentivando la elección de este último por medio de beneficios como destinar parte del pago del tributo al programa de Beneficio Económico Periódico (BEP), lo cual permitirá a la población informal acceder al sistema de protección de la vejez, a un seguro de vida, al régimen de independientes de Cajas de Compensación Familiar y a la afiliación a la Administradora de Riesgos Laborales (ARL).

De acuerdo a lo estipulado en el artículo 905 del Estatuto Tributario Nacional, serán sujetos pasivos a este tributo las personas naturales que tengan establecimientos con actividades comerciales al por menor, como tiendas, con un área inferior o igual a $50 \mathrm{~m} 2$ e ingresos brutos entre 1.400 y 3.500 UVT. El valor anual del impuesto oscila entre 16 y 32 UVT dependiendo del volumen de ingresos del contribuyente.

Una alternativa muy beneficiosa en la medida de que reduce la informalidad, simplifica los trámites tributarios y brinda beneficios tentativos a los contribuyentes, pero son beneficios desapercibidos por la totalidad de tenderos entrevistados, los cuales manifestaron desconocer por completo este tributo; adicional a esto, por ser un impuesto voluntario las personas suelen pasarlo por alto además que el Estado no ha generado una presión o control constante para su aplicación.

\section{II.2.2 Seguridad social}

La afiliación al sistema general de pensiones es obligatoria para todos los trabajadores dependientes e independientes. De igual forma, al régimen contributivo de salud deben afiliarse los trabajadores independientes con capacidad de pago. En general, se establece que "Todos los empleados, trabajadores independientes (con ingresos totales mensuales superiores a 1 SMLMV) y los pensionados, deben estar afiliados al Régimen Contributivo". (Ley 797, 2003, art. 13)

Obligatoriedad que de acuerdo a lo observado en las entrevistas realizadas, no está siendo cumplida por la mayoría de los tenderos entrevistados, sólo tres personas contestaron afirmativamente el aporte a seguridad social, pero argumentando que era aportado por fondos provenientes de trabajos externos a la tienda.

\section{II.3 Sobre las prácticas contables}

La contabilidad como sistema de control e información, se configura en un sistema fundamental que complementa los procesos y las dinámicas que se 
desarrollan dentro de la microtienda, en términos formales, la contabilidad facilitaría la planeación estratégica y ejercer el control de la gestión de forma que, haciendo un uso eficiente de los recursos disponibles se lleve la unidad económica a la posibilidad de competir con sus pares, maximizar sus ganancias y posicionarse en una industria determinada, la microtienda como unidad económica no es muy ajena a lo que sucede en una empresa legalmente constituida, por lo que la contabilidad en escalas menores buscaría el mismo objetivo: controlar los recursos de forma que se dé el potencial para generar utilidades y permanencia en el tiempo.

\section{II.3.1 Contabilidad como requerimiento de ley}

El Decreto 410 de 1971 (Código de Comercio) en sus artículos 10 y 20, enmarca a los dueños de las microtiendas como comerciantes por el hecho de realizar la actividad, característica que los convierte en sujetos pasivos para el cumplimiento de las obligaciones que en el mismo código se estipulan en su artículo 19, entre las que se encuentra llevar contabilidad conforme a la ley; lo que entre otros aspectos asigna la condición de formalidad a los mismos.

Con respecto a lo anterior, la contabilidad a la que se hace referencia, es la establecida en el Decreto 2706 en el 2012 donde se establece entre otras cosas, el marco técnico normativo de información financiera para las microempresas y su periodo de aplicación obligatoria, la cual sigue los lineamientos de la convergencia a estándares internacionales materializados en la Ley 1314 del 2009, y por medio de la cual se establece en su artículo 2, que el Gobierno autoriza a ciertos obligados a llevar contabilidad para que la elaboren de forma simplificada, con estados financieros y revelaciones básicos, adopción que dependería del volumen de activos, ingresos, número de empleados, forma de organización jurídica y circunstancias socioeconómicas.

Es en este momento es preciso mencionar que esa autorización de simplificar la contabilidad, no se realizó acorde a las necesidades reales de las microempresas, sino que por el contrario, fue un proceso de eliminación de obligaciones que aunque algunas fueron racionales, como suprimir el valor razonable, otras no lo fueron, como la eliminación de la obligación de preparar y presentar el estado de flujos de efectivo. En cuanto a este informe, es preciso mencionar que, según lo observado, resultaría más provechoso para la toma de decisiones de las microtiendas, un informe que refleje el dinero disponible, en lugar de uno donde se muestre el valor de la propiedad, planta y equipo. Esto, como consecuencia de que los tenderos cada día cuentan el dinero disponible, para tomar decisiones de presupuestos y reinversión en inventarios al día siguiente; más no se preocupan por conocer el valor del negocio periódicamente. 
Giraldo, Sinisterra, Arteaga. Prácticas contables generalmente utilizadas en el sector informal...

Dejando a un lado la crítica al proceso de construcción del marco normativo simplificado, se continúa mencionando que la razón por la cual los tenderos deberían aplicar la contabilidad simplificada, es por cumplir con los requisitos estipulados en el Decreto 3019 del 2013 y la Ley 1819 del 2016, en donde se establece que para ser categorizado como pequeña empresa, siendo estos, contar con menos de 10 trabajadores, activos totales inferiores a 500 SMLMV, ingresos brutos inferiores a 3.500 UVT.

Aunque ya se identificó las NIIF para microempresas como la regulación contable aplicable al sector investigado, no sobra señalar la normatividad local que abarca, entre otras cosas, la forma de llevar contabilidad y los mecanismos para hacerlo. De acuerdo con esto, el artículo 616 del estatuto tributario establece que las personas pertenecientes al régimen simplificado de IVA, deberán llevar un libro fiscal foliado donde se registren discriminadamente las operaciones diarias del microestablecimiento; y en donde se debe totalizar el valor pagado en compras e ingresado por ventas, de acuerdo a sus soportes. Adicional a esta norma, el Acuerdo 64 de 2012 del Municipio de Medellín, en su artículo 50 parágrafo 1 , establece que los contribuyentes del régimen simplificado de ICA, deberán llevar un libro de registro de operaciones diarias; pero, aunque éstas regulaciones sólo exigen la preparación de los mencionados libros, va en contravía afirmar que bastaría con la preparación de estos, puesto que para efectos del código de comercio, al considerarse los tenderos como comerciantes, deben cumplir con llevar los libros que la ley exija por su condición, incluyendo los contemplados en la NIIF simplificada. Lo anterior para dar a entender que, la obligación de elaborar el libro fiscal o el libro de registro de operaciones diarias, no sustituye la obligación de preparar los demás informes contables exigidos.

En este punto, cabe cuestionarse sobre la efectividad y sustento de la implementación de normas simplificadas a los pequeños negocios, en donde su contenido y exigencia no es acorde al contexto económico y social de estos. Si bien el proceso de globalización e implementación de normas internacionales es una oportunidad para la profesión en el sentido de unificar el lenguaje y prácticas contables a escala mundial, también se cuestiona la forma en que se adoptaron e impusieron dichas normas.

El proceso de globalización implicó la unión de mercados, sociedades y culturas del mundo por medio de transformaciones políticas y económicas que implicaron un carácter global, se dio paso a la era de la información, lo que significó grandes volúmenes de información fluyendo en la atmósfera mundial, además acarreó consigo el desarrollo de innumerables tecnologías. Difícilmente una organización pudo sustraerse de tales cambios por lo que las empresas locales se vieron enfrentadas a nuevos retos, a nuevos procesos que 
les demandaron mejora y eficiencia en sus estrategias, cosa que si no lograban se verían sumergidas en el fracaso.

La contabilidad como el sistema que clasifica, registra, consolida y presenta los hechos financieros realizados en un periodo determinado se convierte en la respuesta a un sinfín de necesidades administrativas, se podría estar hablando de

La economía de la información, entendida como aquella que tiene como concepto rector la eficacia, es decir, proporcionar valor a clientes-objetivo en un marco altamente competitivo. Un enfoque que obliga a orientar la información hacia el futuro para anticipar cambios y con ello oportunidades. (A.C., 1994, p. 35)

En este sentido, la contabilidad se configura como un factor estratégico que complementa la gestión de cualquier entidad o unidad económica, debido a que la información que resulta como producto de la aplicación de un sistema contable a la entidad, sumado a la información no financiera claro está, servirá de base para tomar decisiones que faciliten a la empresa la competitividad, un crecimiento real y rentabilidad; es importante aclarar que, es necesario que tal información se brinde oportunamente para que pueda agregar el valor que merece a las empresas, de otra forma, si esta no es veraz y oportuna, las decisiones pueden tomarse de forma errónea o peor aún, no tomarse.

Enfocando este concepto a las microtiendas, se podría decir que aunque informales en cierto sentido, se envuelven en la misma dinámica de una empresa en la formalidad, en búsqueda de rentabilidad y competitividad frente a otras tiendas en su contexto, para este tipo de establecimientos no es precisa la contabilidad con todo el rigor que la norma supone, pero si es importante y necesario un sistema contable mínimo que permita básicamente el control de los recursos, es decir, de inventario, cuentas por cobrar, proveedores pendientes de pago, efectivo disponible, entre otros, que en general, brinde la información necesaria para el direccionamiento de la unidad de forma que sea rentable para sus dueños o inversionistas.

\section{II.4 Necesidades de información}

La microtienda como unidad económica, se desenvuelve en un macrosistema abierto, definidos estos como aquellos que "reciben influencia del resto del universo, pero solo a través de ciertas vías especificas llamadas entradas (insumos); o que ejercen influencia sobre el resto del universo, pero solo a través de ciertas vías especificas denominadas salidas (productos)" (Bermejo, 1994), es decir que al igual que cualquier otra empresa, la entidades en cuestión deben relacionarse con entes externos a ellos como Proveedores, clientes, Estado, entre otros, en pro de conseguir su objetivo.

Partiendo de este enunciado y evaluando lo mencionado por los tenderos en la aplicación del instrumento, se obtiene que para la mayoría de ellos, la 
Giraldo, Sinisterra, Arteaga. Prácticas contables generalmente utilizadas en el sector informal...

información importante se resume en conocer los ingresos totales del día, el valor de las compras a realizar para surtir el negocio al día siguiente y el detalle de las cuentas por cobrar en los casos que se realiza el coloquialmente llamado "fiado". Inicialmente fue fácil evidenciar que la necesidad de información para una microtienda es mínima, debido a que se halló que en la mayoría de las tiendas se producía poca información relativa a los movimientos y transacciones del negocio, información que, para el manejo y sostenimiento del establecimiento es suficiente, es preciso mantener a la vista que en general, la proyección de este tipo de establecimientos no es crecer o expandirse, sino mantenerse en el tiempo, sobrevivir el día a día, lo cual podría entenderse como una justificación al desinterés del tendero por generar informes contables que le den cuenta de inventarios, ganancias, cuentas por pagar, entre otros.

Retomando lo mencionado en párrafos anteriores, la empresa es requerida por aquellos agentes en su sistema y que se configuran en terceros interesadas en sus utilidades, en su rendimiento, en su eficiencia, en fin en sus resultados, por lo que es necesario el proceso de rendición de cuentas y se da la importancia de la información contable, bien se menciona en la revista de Contaduría de la Universidad de Antioquia que "La contabilidad dejó de tener un solo usuario, el propietario del ente económico, para entrar a ser apreciada por multiusuarios en lo que puede considerarse como una nueva concepción de empresa" (Castaño, Prieto, y Machado, 1997, p. 123-138).

\section{II.5 Explorando las prácticas contables de los tenderos y otros resultados}

Habiendo identificado las distintas necesidades de información contable que de forma general se presentan en las microtiendas, resulta un poco más clara la capacidad de ilustrar las prácticas contables que utilizan para satisfacer las mismas. Sin embargo, es menester realizar una definición que desde el concepto de práctica contable se adecúe al tamaño y al contexto de las microtiendas.

Así entonces, si se define el concepto de práctica contable a través de la interpretación de la definición dada por la Real Academia Española al término de práctica, como un conocimiento que se lleva al hacer dentro de la realidad de manera continuada, en su mayoría diestra y conforme a las reglas que rigen el tema del contexto (Real Academia Esapñola, s.f). Cuando se habla de prácticas contables se puede apreciar este concepto inmerso en el contexto de la contabilidad, es decir, en lo referente al reconocimiento, medición, clasificación, presentación y análisis de hechos económicos que lleva a cabo la entidad, incluso si algunas o todas las actividades que se realicen dentro de estas prácticas se hagan de forma empírica, pero que desde la perspectiva contable se entiendan para estos fines (García, Vásquez, y Zapata, 2016). 
De igual forma, para el desarrollo de dichas prácticas contables son necesarios varios instrumentos de contabilidad que se entienden como todas aquellas herramientas físicas, virtuales y otras, que se utilizan en su ejecución, como ejemplo de ellas se encuentran los libros contables, software contable, kárdex, plan de cuentas, estados financieros, documentos soporte, entre otras. Sin embargo, ésta ejemplificación puede enmarcarse en ámbitos más empíricos y recursivos como cuadernos, informes de carácter más sencillo e incluso el llevar cuentas sin registro alguno sino, en base a la memoria y las relaciones sociales.

En éste sentido, si se permite abrir un poco la mentalidad respecto a los preceptos teóricos y técnicos que tiene la contaduría pública, y se enfoca la atención más en su propósito que en su amplio desarrollo conceptual, pues se podrá dar la connotación de práctica contable a todas las formas en las cuales los tenderos captan la información de las distintas transacciones económicas, la entienden y la reexpresan en las decisiones que toman respecto a su negocio. Al hablar de formas, cabe resaltar que son múltiples los hallazgos acerca de éste tema; los tenderos no tienen la necesidad de tener muchos libros con registros para entender el funcionamiento y las necesidades de su establecimiento; pueden pronosticar los resultados de la microtienda en un período, por su historial y promedio mental de ventas, costos y gastos; además de que podrían tener una noción empírica aplicada de lo que es la rentabilidad y la valoración del negocio.

Éstas formas en las cuales no se realiza énfasis en tener soportes físicos ni desarrollos documentales históricos ni presupuestales, también podrían deberse a que las microtiendas no basan su funcionamiento en el principio de negocio en marcha que define que se garantice la continuidad del negocio teniendo en cuenta su capacidad de adquisición permanente del nivel de ingresos suficientes para cumplir sus obligaciones, el cual es uno de los fundamentos principales de la contabilidad de devengo actual (Molina, 2014). Desde la perspectiva de los tenderos, las tiendas son creadas para su sostenimiento, quizás la permanencia de las mismas, pero no para su constante crecimiento; esta aclaración ha de respaldarse con las afirmaciones recurrentes entregadas por los tenderos: "Si esto no está dando, se cierra y ya" o "si no estuviera dando, no la tendría".

Ya que se está realizando una interrelación de conceptos entre lo ideal y lo más empíricamente aplicado, se pueden identificar entonces varias asociaciones a lo largo del acercamiento al conocimiento de los tenderos y su cotidianidad, enfocando las mismas en las prácticas contables que usualmente se definen desde el quehacer profesional y que se desarrollarán a continuación.

Se tiene el reconocimiento como fase o práctica inicial, la cual se refiere desde el marco técnico de contabilidad a la incorporación de los elementos de 
Giraldo, Sinisterra, Arteaga. Prácticas contables generalmente utilizadas en el sector informal...

las transacciones económicas a los estados financieros bajo unos parámetros establecidos (Molina, 2014), respecto a la cual se propone una adaptación para el contexto microeconómico estudiado en la que se puede definir como la aprehensión que tienen los tenderos frente a las distintas transacciones que realizan en su cotidianidad, que según lo referido por los mismos, son vender de contado o fiar, comprar, guardar del efectivo lo destinado al pago de mercancía y gastos como arriendo y servicios, al igual que la inversión en el mejoramiento del negocio y guardar una utilidad que reflejan ya sea como salario o gastos personales. Mediante la interlocución con ellos, se identifica que por la consciencia de todos estos eventos, comprenden el concepto empírico de lo que son derechos y obligaciones en términos económicos y aunque no hagan clasificación de los mismos mediante las categorías de activo, pasivo, patrimonio o las cuentas de resultado, sí tienen una percepción de qué y cuánto es de su propiedad y lo que corresponde a terceros.

Es decir, en la práctica contable concerniente a la clasificación la cual comprende la distribución de la cuantía de las transacciones económicas en categorías que bajo el estándar contable deberían cumplir con el criterio de partida doble, los tenderos no llevan registros económicos bajo estas características, pero por la misma consciencia del haber y deber descrita anteriormente se podría entender que guían su actuar y el manejo de los recursos bajo una racionalidad económica y contable.

Para ejemplificar lo enunciado se tiene entonces, la aplicación de instrumentos contables que desde la adaptación empírica se pudieron observar, tales como el principal cuaderno de cuentas por cobrar "Fiados", el resguardo de las facturas de proveedor de las cuales establecen el ahorro o la destinación de efectivo para tal fin, un arqueo de caja de manera diaria y la distribución del dinero para obligaciones personales (utilidad), pago de proveedores y ahorro. Algunos poseen un libro donde registran los resultados diarios, y ocasionalmente hay quienes usan el libro tradicional de contabilidad. Dichas herramientas tienen características de la contabilidad que sirven para su propósito esencial y dan una especie de producto suficiente para el control de los recursos desde la perspectiva del tendero, aunque las mismas no tengan el carácter técnico y probatorio del "deber ser" fundado por el marco técnico de la contaduría pública y las normas de fiscalización de las administraciones tributarias.

Desde la práctica contable enmarcada en el término de medición, definida como el proceso de cuantificación del importe por el que se expresarán los elementos de los estados financieros (Molina, 2014), se encuentra que los tenderos por realizar la transacciones económicas con una perspectiva de contabilidad de caja, es decir, lo efectivamente realizado o pagado en su momento y cuyos gastos ya tienen una cuantía definida, asume dicha 
cuantificación por lo que sería el costo histórico, es decir que el valor de sus bienes, ventas, inventarios y gastos serán los estipulados en las respectivas facturas, sin ningún tipo de distinción entre lo que son los costos, impuestos o demás ítems que usualmente se desglosan en la contabilidad formal.

Otros aspectos relacionados con esta práctica y que amplían un poco su aplicación, se evidencian con cuestionamientos realizados sobre la plusvalía y los precios de venta que determinaban los vendedores en su microestablecimiento. De esta forma un inciso relacionado, sería la valoración que cada tendero establecía para su negocio cuando se consultaba por cuánto lo venderían, para esto, ellos tienen en cuenta su inversión inicial, la mercancía existente, los refrigeradores y estanterías y aunque no la mayoría de las personas, varios tuvieron presente el nivel de ventas usuales (lo que se considera flujos de caja proyectados) y el punto comercial de su establecimiento al igual que la clientela conseguida. Adicional a este ejemplo, también para la estimación del precio de venta de la mercancía, se identificó que existen tres métodos usuales que son acatar el precio sugerido por el proveedor, la aplicación de un porcentaje definido entre el $15 \%$ y el $25 \%$ sobre el precio de compra, y por último la comparación de precios entre competidores cercanos.

Los aspectos relacionados en el párrafo anterior, son un ejemplo consistente de que, aunque los conocimientos contables y económicos de los tenderos no sean muy técnicos, su corte empírico no está del todo aislado de varias de las prácticas de medición y valuación realizadas dentro de los establecimientos con contabilidad formalizada y técnica, sino que las mismas se atañen a la facilidad que es necesaria en el contexto.

Como una de las prácticas finales se encuentra la presentación, en la que se observa una de las mayores diferencias entre la contabilidad de las grandes empresas y los microestablecimientos en cuestión; se identifica que los tenderos no tienen un especial interés en la obtención de informes periódicos ni resultados consolidados de las distintas transacciones que se realizan. Su posición se sostiene en el concepto de que estos documentos tienen el objetivo de rendir cuentas a terceros y al ellos trabajar de forma personal o en compañía de sus familias, dichos procesos de información no son necesarios.

Frente a este aspecto se podría plantear la argumentación de que los informes o estados financieros no tienen como única característica su capacidad probatoria, sino que con base en estos se pueden realizar análisis y por ende tomar decisiones, pero en el contexto cotidiano de las microtiendas, la población estudiada mostró un factor común respecto a las formas en las que basan su actuar, como es el conocimiento empírico y mental del funcionamiento de su negocio y la situación real del efectivo disponible. 
Giraldo, Sinisterra, Arteaga. Prácticas contables generalmente utilizadas en el sector informal...

Es decir, según su percepción, los informes requieren de mucho tiempo en su elaboración y no presentan mayor utilidad práctica, pues ellos establecen que conocen el promedio de los resultados obtenidos y al no tener una proyección amplia de reinversión, las decisiones de compra de mercancías, la obtención de utilidad y la consecución de préstamos, se toman con la capacidad de adquisición correspondiente al efectivo disponible y la conciencia de la cuantía de gastos fijos (arriendo y servicios públicos) para el período.

Al haber realizado la identificación de las prácticas contables de reconocimiento, clasificación, medición, presentación y análisis, en el contexto económico de las microtiendas se intentó realizar un análisis muy similar al aplicado cuando se fundamentaron los principios de contabilidad generalmente aceptados, para los cuales se asoció la opinión, prácticas e instrumentos de una gran cantidad de contadores o personas encargadas de llevar a cabo las labores contables, para de esta forma encontrar las mejores formulaciones y bases teóricas y prácticas del quehacer contable. Relacionado a esto se ha sustentado que los instrumentos de registro y manejo de la información contable aparecen no como un requerimiento nacional de un Estado, sino como un cuerpo de costumbres y prácticas de una "clase social" (Casal y Peña, 2005) lo que en nuestro caso específico de estudio serían los tenderos.

Desde otra arista de la investigación, de acuerdo con lo evidenciado en el trabajo de campo, la mayoría de tenderos sólo cumplen con lo exigido en el Acuerdo 64 de 2012 del Municipio de Medellín, es decir, con el registro diario de sus operaciones, resaltándose un cumplimiento parcial, en la medida en que la tendencia es llevar control de las ventas, mas no, de las compras, devoluciones o pérdidas que se puedan presentar. Esta actuación no está fundamentada en la intención de evadir sus obligaciones, sino de suplir una necesidad de información que resulta ser útil y suficiente para la continuidad de su negocio.

Con respecto a lo anterior y abordando un poco el contexto externo, se hizo fácil comprobar que en general las microtiendas son concebidas por una sola persona, su dueño, así que no se requiere la rendición de cuentas a inversionistas, los clientes sólo buscan la satisfacción de necesidades a través de productos o servicios por lo que no están muy interesados en conocer el estado financiero y económico de quién les provee. Por otra parte, el Estado, desde los entes territoriales y entidades públicas, se configura en el único sujeto externo que directa o indirectamente solicita información de las microtiendas para efectos de ejercer control, fiscalización y tributación, esto fue evidente en el hecho de que si no todas, la gran mayoría contaban con registro en Cámara de comercio y la Dian, y como consecuencia tributaban lo correspondiente. En lo relacionado con el reporte de información a proveedores, enfocado a si estos últimos, para acceder como tales requieren información como inscripciones 
legales, o informes que den cuenta de la situación del microestablecimiento, es un tema que queda en el tintero debido a que no se abordó en la investigación, pero que no afecta el adecuado desarrollo de ésta.

\section{Llamado de atención a la contabilidad}

A lo largo de esta redacción, frente a la implicación que tiene la contabilidad para las empresas y la contextualización de las microtiendas en los ámbitos comercial, económico, financiero y legal, donde se ponen en manifiesto las diversas necesidades de información que se podrían tener y satisfacer en gran medida a partir del quehacer contable; se debe reconocer que la contabilidad estructurada tal y como se presenta actualmente en el país como lo es el marco técnico normativo contable para microempresas, puede no satisfacer dichas necesidades sin implicar un alto costo no sólo de los equipos y documentación, sino también de la capacitación de los tenderos o el pago del personal que realice dichas tareas.

Esta posible problemática da pie para entender que en las microtiendas se implementan medios empíricos alternativos para el manejo de sus recursos, y que pueda que éstos no garanticen una información que supla todas las características probatorias, pero son instrumentos que hasta el día de hoy han sido útiles para mantener en marcha el microestablecimiento. En contraste, al cuestionarse por contenidos en materia de teoría y de desarrollos contables acerca de instrumentos idóneos, es decir, de acuerdo a las capacidades y necesidades de un sector tan relevante como es éste en la economía nacional, no son de fácil hallazgo o interpretación.

A partir de la identificación de las necesidades de información y prácticas de carácter contable de mayor aceptación por parte de los tenderos, se busca acudir al interés de profesionales y estudiantes de la ciencia social que es contabilidad, para lograr un desarrollo que además de acoplarse a los requerimientos de la población objeto de la investigación, logre reflejar información requerida por los entes gubernamentales con el fin de aportar en la formalización y permanencia de las microtiendas.

En concordancia con el tema de la formalización y de las prácticas contables que esto implica, se observa que el gobierno ha intentado plantear desde sus decretos y leyes un ámbito más asequible, mediante el decreto 2706 del 2012 conocido como el Decreto Único Reglamentario y cuya función es reglamentar el marco técnico de información financiera para las microempresas. A través de éste, se ha pretendido sentar las bases de una contabilidad simplificada con lenguaje menos técnico y con menor número de responsabilidades.

Se plantea entonces, que se debería lograr un equilibrio entre la disposición del tendero, la regulación gubernamental y la contabilidad, con el fin potenciar este sector de la economía que ha sido de tanta trascendencia en el país y 
Giraldo, Sinisterra, Arteaga. Prácticas contables generalmente utilizadas en el sector informal...

facilitar desde el conocimiento que atañe a los contadores, el bienestar social, puesto que teniendo bases para el diseño de instrumentos de fácil manejo para los tenderos se puede incrementar además de la productividad, el interés de formalización y por ende el pago de impuestos, que resulta en un bienestar común.

\section{Discusiones adicionales}

- Siendo las microtiendas unidades económicas con una participación importante en la economía nacional respecto a su generación de empleo y expansión de la actividad comercial, se considera que carecen de la atención que éstas requieren, tanto por parte del Estado como de los de Contaduría Pública en lo referente a la adaptación de sus respectivos marcos regulatorios y técnicos al contexto en el que se desenvuelven.

- Aunque hay marcos técnicos contables que según sus parámetros son aplicables a las microtiendas, se considera que no están alineados a las condiciones y necesidades propias de las mismas, es decir que es muy extensa la relación costo-beneficio, es decir, que se va en contravía al principio de la economía de la información y que por otra parte, adaptar un marco técnico a los microestablecimientos no es amputar todo un proceso como lo es el estado de flujos de efectivo, sino simplificar tareas que son necesarias y eliminar otras tantas que no lo son y adicionar las que hagan falta.

\section{Conclusiones}

Si bien las microtiendas cumplen con varios de los requerimientos legales básicos como la matricula en el registro mercantil y la expedición del RUT, dejan de cumplir deberes formales como llevar contabilidad en debida forma y el pago de seguridad social, situación que les categoriza en el sector informal.

Las principales necesidades de información de los tenderos identificadas en el trabajo de campo y que para su labor consideran que tienen satisfactores suficientes, son la cuantía de las ventas diarias, el control de los deudores y las compras a realizar el día siguiente, con la intención de programar los ingresos y desembolsos de efectivo y establecer la utilidad neta. Por otra parte, no realizan provisiones ni ahorros para posibles contingencias como se realizan en las empresas más grandes.

Para suplir las necesidades previamente enunciadas, son utilizados comúnmente en la población estudiada, mecanismos o instrumentos contables empíricos, tales como cuaderno de deudores, resguardo de las facturas de proveedor, arqueo de caja de manera diaria y la distribución del dinero para obligaciones personales (utilidad), pago de proveedores y ahorro; algunos 
poseen un libro donde registran los resultados diarios, entre otros, los cuales son de uso inmediato, y no suelen guardarse por un tiempo prolongado.

Finalmente, los instrumentos previamente enunciados son las herramientas a través de las cuales se llevan a cabo las que si asimilaron como prácticas contables generalmente realizadas en las microtiendas, que respecto al reconocimiento y clasificación se identifica que comprenden el concepto empírico de lo que son derechos y obligaciones en términos económicos y aunque no hagan clasificación de los mismos mediante las categorías de activo, pasivo, patrimonio o las cuentas de resultado, si tienen una percepción de qué y cuánto es de su propiedad y lo que corresponde a terceros. En lo referente a la medición, el costo histórico es su principal criterio, y también se observa que consideran el valor agregado respecto a la valoración de su establecimiento y la determinación del precio de venta. En cuanto a la consolidación periódica y presentación de informes, no se tiene un patrón definido para la generación de informes ya que no existe una necesidad interna latente de estos, debido a que aun no existiendo prácticas muy formales de contabilidad, existe una contabilidad en la cabeza del tendero basada en su experiencia, sobre la cual se toman las decisiones de reinversión, gastos y utilidad a obtener, lo cual permite percibir que comparte criterios similares a la idea de la contabilidad como racionalidad calculadora de capital.

En esta línea, cabe resaltar el sentido de la presente investigación respecto al intento de conocer y documentar las necesidades de información y prácticas contables resolutivas que aparecen como mera intuición entre los conocedores del área y se dan como hechos, o a los que no se les otorga importancia con justeza siendo estos cotidianos en la sociedad.

\section{Referencias bibliográficas}

A.C., I. m. (1994). El contador público en la era de la información. . México : Instituto mexicano de contadores públicos de México. A.C.

Abreu, J. L. (2012). Hipótesis, Método y Diseño de Investigación. International Journal of Good Conscience, 187-197.

Banco de la República. (2015). Biblioteca virtual Luis Ángel Arango. Obtenido de Oferta

y demanda: http://www.banrepcultural.org/blaavirtual/ayudadetareas/economia/ oferta_y_demanda

Bermejo, J. C. (1994). Entre historia y filosofía. Madrid, España: Akal.

Casal, R. A., y Peña, A. E. (2005). Principios Contables. Mérida, Venezuela.

Castaño, O. L., Prieto, E., y Machado, M. A. (1997). La información contable frente a las necesidades del usuario. Contaduría Universidad de Antioquia, 123-138.

Congreso de la república. (13 de Julio de 2009). Convergencia a NIIF. Ley 1314. Colombia. Congreso de la república. (29 de Diciembre de 2016). Ley 1819. Reforma Tributaria. Colombia. 
Giraldo, Sinisterra, Arteaga. Prácticas contables generalmente utilizadas en el sector informal...

DANE. (22 de Marzo de 2017). Boletín Técnico Microestablecimientos . Obtenido de Departamento Administrativo Nacional de Estadística: https:/www.dane.gov.co/files/ investigaciones/boletines/microestablec/Bol_micro_2016.pdf

Días, M. (20 de Marzo de 2014). Tiendas de barrio jalonan la economía Colombiana. Obtenido de El espectador: http://www.elespectador.com/noticias/economia/tiendas-de-barriojalonan-economia-colombiana-articulo-481374

García, J. F., Vásquez, A., y Zapata, E. M. (2016). La practica contable de principios del siglo $\mathrm{XX}$ en el ferrocarril de antioquia y la gobernación de antioquia. Adversia, 4.

Montoya, J. (2004). El discurso historiográfico de la sociedad económica de amigos del estado de Guatemala en la primera mitad del siglo XIX. Primeros acercamientos desde la historia cultural. ECA, estudios centroamericanos, 87-118.

INDESCO. (2003). Plan para iniciar un proceso de desarrollo a través de la economía solidaria . Revista Cooperativismo y Desarrollo (Universidad cooperativa de Colombia), 111119.

Ministerio de comercio, industria y turismo. (27 de Marzo de 1971). Código de comercio. Decreto 410. Colombia.

Ministerio de comercio, industria y turismo. (27 de Diciembre de 2012). Decreto 2706. Marco técnico normativo de información financiera para microempresas. Colombia.

Ministerio de comercio, industria y turismo. (27 de Diciembre de 2013). Decreto 3019. Modificación al decreto 2706. Colombia.

Molina, J., y Restrepo, M. (2012). Informalidad y economía popular: las ventas ambulantes en el centro de Armenia 2009-2010: Actividad económica, su importancia en la reproducción ampliada de la vida y generación de excedente de capital. Bogotá: Ediciones Grancolombianas.

Molina, H. (2014). Orientaciones técnicas NIIF-Marco Conceptual. Colombia: Consejo Técnico de Contaduría Pública.

Moreno, S., y Colorado, P. (1997). Economía popular y circuitos económicos. Controversia , 70-82.

Ramírez, J. C., y Vergara, G. A. (2014). Oportunidades, sostenibilidad y riesgos de los negocios informales de la comuna diez de Cali. (U. d. Cali, Ed.) Guillermo de Ockham, XII(1), 35-42.

Razeto Migliaro, L. (2003). La economía popular de solidaridad en una perspectiva de desarrollo alternativo. Revista Cooperativismo y Desarrollo (Universidad cooperativa de Colombia), 57-62.

Real Academia Esapñola. (s.f). Diccionario de la Real Academia Española. Prácticas. 